\title{
Uso de las TIC para proyectos productivos en las instituciones educativas del Municipio Zona Bananera. Magdalena. Colombia
}

\section{Use of ICT for productive projects in the educational institutions of the Bananera Zone Municipality. Magdalena. Colombia.}

\author{
Carlos Lattá Arias \\ lattac@yahoo.es \\ Institución Educativa Departamental Las Mercedes \\ Municipio Zona Bananera. Colombia \\ https://orcid.org/0000-0001-8461-1914
}

Recibido: 02 de octubre del 2018

Aprobado: 02 de noviembre del 2018

\begin{abstract}
RESUMEN
La investigación tuvo como finalidad analizar el uso de las tic para proyectos productivos en las instituciones educativas del Municipio Zona Bananera. Magdalena, Colombia. Sustentado bajo teorías de: Guzman y Briceño (2010) Orfebre (2011) Soto Márquez (2010), entre otros. Enmarcado bajo el paradigma positivista, bajo un enfoque empírico -inductivo. Tipo de investigación descriptiva analítica. Bajo un diseño no experimental, transeccional de campo. La población sumatoria de los estratos o subconjuntos que representan la muestra de ciento cuarenta y dos (142) sujetos pertenecientes a las Instituciones educativas en estudio. Las técnicas e instrumentos de recolección fueron conformados por preguntas de tipo alternativas de respuestas cerradas y múltiples. La confiabilidad fue de 0,858 . Se revelaron debilidades en directivos y docentes, los indicadores tienen poca presencia en la dimensión capacidades tecnológicas, concretamente con el manejo de programas, aprovechamiento de la información y manejo del internet, siendo desfavorable para impulsar proyectos en correspondencia con las necesidades de autogestión y de aprendizaje en los estudiantes.
\end{abstract}

Descriptores: Uso de las tic; proyectos educativos; capacidades tecnológicas; autogestión; aprendizaje.

\section{ABSTRACT}

The purpose of the research was to analyze the use of tic for productive projects in the educational institutions of the Zona Bananera Municipality. Magdalena, Colombia 
Sustentado under the theories of: Guzman and Briceño (2010) Orfebre (2011) Soto Márquez (2010), among others. Framed under the positivist paradigm, under an empirical -inductive approach. Type of analytical descriptive investigation. Under a nonexperimental, transectional field design. The summing population of the strata or subsets that represent the sample of one hundred and forty-two (142) subjects belonging to the educational institutions under study. The collection techniques and instruments were shaped by alternative questions of closed and multiple answers. The reliability was of 0.858 . Weaknesses were revealed in managers and teachers, the indicators have little presence in the dimension of technological capabilities, specifically with the management of programs, use of information and internet management, being unfavorable to promote projects in correspondence with the needs of self-management and learning in the students.

Descriptors: Use of tics, educational projects, technological capabilities; selfmanagement learning.

\section{INTRODUCCIÓN}

Las tecnologías de la información y comunicación, denominadas TIC, han arropado a todas las actividades realizadas por los seres humanos, desde las de orden cotidiana, hasta las de índole laboral, científico, deportivas, culturales, lo que ha conllevado a capacitarse en ese sentido, y hacer de las mismas unas herramientas de trabajo efectivas, en beneficio de las actividades que se realizan.

Por lo tanto, la incursión de las Tecnologías de la Información y la Comunicación de carácter digital en la cotidianidad de los individuos, ha generado cambios en diferentes ámbitos de la sociedad: las relaciones interpersonales, forma, posibilidad de acceso al conocimiento, el acceso instantáneo a la amplia oferta de información online, las nuevas configuraciones económicas, políticas, el fenómeno de la globalización que cada vez se fortalece, toma más forma en la denominada sociedad de la información y la comunicación.

En este sentido, las instituciones educativas de Latinoamérica, buscan la satisfacción de las necesidades propias de los docentes, empleando las TIC, por ser un soporte para la gestión del conocimiento, siendo este un factor para el logro de los objetivos organizacionales. Este orden de ideas, las tic, son indispensable en la vida de la 
institución educativa, por facilitar el acceso a fuentes de información, haciendo más rápido el trabajo, la posibilidad de trabajar en equipos, creando un pensamiento sistemático, el acceso a las fuentes de la información, sean mayores a lo que el trabajo se realiza en la institución.

De igual manera, las TIC, pueden contribuir al acceso universal en educación, igualmente a la instrucción de ejercicio sobre la enseñanza-aprendizaje, al igual que la calidad de desarrollo profesional en los docentes, así como, la supervisión y gestión más eficientes del sistema educativo, e incluso esas tecnologías han impactado sobre la experiencia humana entorno al abordaje de nuevos conocimientos en la sociedad.

Ahora bien, en la sociedad educativa actual, la falta de la integración de la escuela con la comunidad, que se ha observado a través de los años, es la base fundamental del problema planteado, debido a que la comunidad, debe asumir su responsabilidad en la calidad de educación que reciben sus ciudadanos, donde la escuela ejerce su deber en convertirse en centro para la transformación de la sociedad, abrir el espacio para el quehacer comunitario, generando propuesta y proyectos, que busquen mejorar el entorno donde desarrolla sus actividades.

Ante lo expuesto, se necesita una escuela que genere nuevas estrategias para integrar a la comunidad e involucrarla en sus actividades diarias, logrando una participación real en este contexto, a través de organizaciones de base, como son los consejos comunales, la cooperativas, las organizaciones no gubernamentales (ONG), entre otras, en la gestión escolar que facilite el desarrollo de programas y proyectos, con el fin de promover cambios en el mejoramiento de la institución y de la comunidad en general.

Es por ello, que en el presente estudio se estudia el uso de la tecnología para realizar proyectos productivos, como estrategia metodológica para incorporar la comunidad y a las instituciones educativas del Municipio Zona Bananera del Departamento del Magdalena. Colombia. 


\section{FUNDAMENTACIÓN TEÓRICA}

Para el desarrollo de los fundamentos teóricos de esta investigación, se consideró conveniente estudiar una serie de aspectos relevantes al uso de la tecnología para realizar proyectos productivos, por lo que se hace necesario establecer claramente la correspondencia entre algunos conceptos referidos a las variables; con el fin de construir sustentos teóricos que le de concreción a estos términos, lo cual e indispensables para una mejor comprensión de los mismos:

\section{EL USO DE LAS TIC PARA PROYECTOS PRODUCTIVOS}

Al referirse al uso de las tic se encuentra la definición expuesta por Martínez (2008), señala que consiste en la separación existente entre las personas comunidades, estados, países que utilizan las tecnologías de la información y comunicación como una parte rutinaria de su vida diaria, aquellas no tienen acceso a las mismas y aunque lo tengan no saben cómo utilizarla. De igual manera, la definición más amplia del uso de la tecnología, no solo se involucra el acceso a las tecnologías de la información y comunicación, sino el uso se da de ellas de manera que estas pueden impactar positivamente en la vida cotidiana.

En referencia Oyarzo (2010), señala que el uso de las tecnologías separa los que están conectados a la revolución digital de las tecnologías de la información y comunicación, no tienen acceso a los beneficios de las nuevas tecnologías. En ocasiones la brecha se produce tanto acceso a los beneficios de las nuevas tecnologías, así como, dentro de las comunidades, debido a la gente a uno u otro lado de las barreras económicas y de conocimientos.

De igual manera, Guzman y Briceño (2010), señala que el uso de la tecnología tiende a perpetuar exacerbando las existentes desigualdades de relaciones jerárquicas de poder, estas voces críticas subrayan que los estudiantes acceden a las tecnologías de la información y comunicación como consumidores, no como creadores. 
Ahora bien, en referencia a los proyectos productivos, de acuerdo con Rey (2015), es considerado como una ordenación de actividades y recursos, que se realizan con el fin de producir algo, ya sea bienes o servicios, siendo capaces de satisfacer necesidades o resolver problemas, asimismo sirve para producir bienes, saberes y conocimientos necesarios para mejorar la vida.

Por otra parte, Márquez (2010), considera que los proyectos productivos, son aquellos que impulsan el desarrollo de actividades generadoras de bienes y servicios útiles a la comunidad, enmarcándose dentro del concepto de desarrollo endógeno, generando redes productivas para el desarrollo de un bien común. Asimismo su carácter deberá tener como finalidad no generar ganancias, que enriquezcan a un individuo, sin garantía, la producción de bienes y servicios sin explotar a los involucrados en el proceso productivo, garantizándoles una calidad de vida digna y la satisfacción de las necesidades colectivas.

Igualmente, Orfebre (2011) señala que un proyecto se puede iniciar o crear con base a una necesidad, que se quiere cubrir o en alguna idea específica, para que se considere productivo, debe cubrir esta necesidad o idea de la mejor manera. Obteniendo resultados medibles, que tenga un impacto positivo. De igual manera, el proyecto productivo se encuentra la obtención de productos y servicios con un el fin claramente productivo.

Es por ello que, en las instituciones educativas los docentes deben propiciar los proyectos productivos por ser aquel que está orientado a transformar cualquier materia prima, para convertirla en un producto terminado, con el propósito de satisfacer las múltiples necesidades de bienes y servicios de la población. El uso de las tic para proyectos productivos, tiende a perpetuar las relaciones jerárquicas de poder, en cuanto los estudiantes acceden a las tecnologías de la información y comunicación, en los proyectos productivos para cubrir una idea especifica que se considera productivo, y así mejorar y obtener los resultados que se encuentran en la obtención y servicios con un fin productivo. 


\section{Estratégicas del uso de las TIC}

En referencias a las actividades estratégicas, Márquez (2010), son aquellas que le permiten al docente, desempeñar conocimientos y habilidades específicas sobre cualquier materia de especialidad, así como los métodos, los equipos, y las tecnologías para la enseñanza - aprendizaje Le facilitarán el trabajo y entenderá mejor lo que se espera del rol que desempeña en el proceso educativo.

De acuerdo con, Aldape (2008), el docente requiere tener una visión sistemática para entender la institución como un todo, percibiendo el impacto de sus decisiones y acciones en los diversos subsistemas, en el sistema total, debido a las concepciones entre distintos departamentos, entidades o elementos. Es importante que el docente, sea capaz de detectar cualquier obstáculo, este interfiriendo en el proceso educativo, de analizar diferentes opciones conducentes y de corregir aquello que se encuentre directamente relacionado con su remoción, ya sea el mismo tome parte en la solución con quien responda.

De igual manera, Suarez (2009), manifiesta el docente tendrá además que tomar decisiones, elegir alternativas de acción, acorde a su autoridad y responsabilidad dentro del sistema. Al hacer esto, tendrá que considerar de manera global los objetivos y estrategias de la institución y su rol e impacto en todos los ámbitos de las diferentes áreas la componen.

\section{Capacidades tecnológicas}

Las capacidades tecnológicas y el uso de la tecnología de la información y comunicación, están siendo utilizadas por los docentes actualmente para apoyar la pedagogía para mejorar la práctica en los estudiantes. Tal como lo señala Luthans, (2006), resaltan que se debe explicar el gran impacto que asume el mundo actual, sus nuevas herramientas como son las TIC, estás inducen a una profunda revolución en todos los ámbitos sociales y que afecta además, y principalmente en el mundo educativo. 
En tal sentido, Márquez (2010), señala que se está en frente a una nueva cultura que asume nuevas formas de observar y entender el entorno donde se desenvuelve, que da la oportunidad a nuevos sistemas de comunicación interpersonal de gran alcance, que informa de cualquier actividad que pretendas investigar, y además que proporciona medios para viajar con mayor rapidez y a cualquier lugar que desees, y que presenta nuevos valores y normas de comportamiento.

De igual manera, Fernández, (2009) considera que, es evidente que el uso de las TIC en los docentes permite un gran compromiso para la educación a todos los niveles, principalmente a los estudiantes de educación primaria que están en una constante formación, se debe estar atento. La necesidad de formar nuevos ciudadanos se proyecta de ir más allá de los primeros estudios de profesión que existen a lo largo de la vida. La formación continua es indispensable por las exigencias de los cambios que proporcionan la sociedad actual.

Siendo las funciones de los docentes de hoy totalmente diferente a los de hacen cien años atrás, asumiendo nuevos retos ante una sociedad que les es fácil el acceso al uso de un computador y a una red social que permite información relevante del mundo, donde necesariamente, se exige múltiples competencia procedimentales en los docentes en cuanto a la valoración y selección de información. Es por ello, que las TIC en los docentes, les permite obtener nueva herramientas y usar estrategias para la resolución de problemas y generar trabajos en equipo.

\section{Políticas públicas del uso de las TIC}

De acuerdo con Cadenas y Ruiz (2007) las políticas públicas, son el conjunto de actividades de las instituciones de gobiernos, actuando directamente a través de agentes, que van dirigidas a tener una influencia determinada sobre la vida de los ciudadanos, por ello son consideras como procesos decisionales, un conjunto de decisiones que se llevan a cabo a lo largo de un plazo de tiempo.

De igual manera Lahera (2008) expresa que una política pública, corresponde a cursos 
de acciones, flujos de información relacionada con un objetivo público definido en forma democrática, que son desarrollados por el sector público, frecuentemente con la participación de la comunidad y el sector privado. Es decir, una política pública incluirá orientaciones o contenidos, instrumentos o mecanismos, definiciones con modificaciones institucionales y la previsión de sus resultados.

Por otra parte, Villareal (2010), considera que los cambios que se efectúen en las instituciones formales, no siempre van al mismo ritmo de las transformaciones en las instituciones informales, las reglas formales pueden cambiarse de un día para otro, pero las instituciones informales que se expresan en la cultura de una sociedad cambian lentamente, no siempre en el mismo sentido y dirección de las reglas formales.

\section{EVIDENCIAS METODOLÓGICAS}

La investigación presentada está enmarcada bajo el paradigma positivista cuantitativo, el cual es conocido como aquel donde son recogidos y analizados ciertos datos sobre las variables en función de determinar el grado de asociación o correlación entre las mismas. En este contexto, Barrantes (2002) citado por Cadenas (2011) señala que el paradigma tiene como propósito darle respuesta a una interrogante causa-efecto, buscando predecir la posible conducta futura sobre la base actual.

El modelo epistemológico, se refiere a la representación que se tiene del conocimiento sobre los eventos, ideas, los hechos, que tiene cada contexto o cultura y crea su actividad interpretativa para las ciencias sociales; el paradigma positivista, es el modelo dominante, que descubre, explica, controla y predice una realidad. De acuerdo con, Hernández, Fernández y Baptista (2014), expresan que la idea central de la filosofía positivista sostiene que fuera del ser humano existe una realidad totalmente realizada, acabada y plenamente externa y objetiva que nuestro aparato cognoscitivo es como un espejo que se refleja dentro de sí.

El paradigma de la presente investigación, es el positivista, según Ander-Egg (2004) este cobra fundamento en el postulado "la exigencia de la realidad" la cual expresa el 
rechazo de toda proposición cuyo contenido no tenga correspondencia alguna con hechos constatados. En este sentido, la investigación se apoyará en presupuestos teóricos científicos con el propósito de validar toda acción demandada en la investigación.

En cuanto al enfoque metodológico, es el empírico - inductivo, que de acuerdo con Hernández, Fernández y Baptista (2014), plantean que a través del enfoque inductivo se analizan solo casos particulares, cuyos resultados, son tomados para derivar conclusiones de modo general. Lo que quiere decir, que a través de las observaciones sistemáticas de la realidad se descubre la generalización de un hecho y una teoría. Con las que posteriormente, se establecerán contrastes con las teorías existentes del fenómeno afrontado.

En el mismo sentido, toda investigación debe responder a una perspectiva teórica metodológica; es decir la forma de abordar el objeto de conocimiento, los procedimientos generales y técnicas utilizadas en el proceso de demostración y comprobación de una teoría científica, a bien de ofrecer soporte científico a la producción del conocimiento, derivando con ello la aplicación del método científico.

Lo expuesto anteriormente sirve de fundamento para enmarcar el tipo de investigación utilizada para abordar la variable: Uso de las TIC para proyectos productivos. En este sentido, la investigación, de acuerdo con los objetivos establecidos, se ubica como descriptiva - analítica.

La investigación descriptiva tiene como objetivo central lograr la descripción o caracterización del problema de estudio dentro de un contexto particular. Estos estudios se caracterizan por la búsqueda específica de las propiedades, características importantes de personas, comunidades, grupos en una situación concreta, describiendo el desarrollo o los procesos de cambio existentes a lo largo de toda la investigación.

Al respecto, Hernández, Fernández y Baptista (2014) señalan que el propósito fundamental de los estudios descriptivos se centra en medir, evaluar o recolectar datos sobre diversos conceptos (variables), aspectos, dimensiones o componentes del 
fenómeno a investigar. Estos son útiles para mostrar con precisión las dimensiones de fenómeno o situación, en el caso específico de esta investigación.

De igual forma, Hurtado (2010) señala que los estudios analíticos tienen como objetivo analizar un evento y comprenderlo en término de sus aspectos menos evidentes. En efecto, el presente estudio destaca las características definitorias del objeto de estudio, para analizarla en su comportamiento particular, extrayendo sus componentes estructurales, así como capacidades tecnológicas de directivos rectores y personal docente.

Cabe señalar, que toda investigación debe orientarse por un plan que permita al investigador obtener información precisa, sobre la base del cual se puedan establecerse conclusiones. En este sentido, el diseño de la investigación, cobra un papel importante en este recorrido, puesto que en ella se concretan dimensiones referidas a las propiedades de las variables que intervienen directamente en el contexto, así como, por su temporalidad y la espacialidad que demarca el territorio, donde convergen los fenómenos investigados. Es así, que el diseño de la presente investigación se considera como no experimental, transeccional y de campo.

Con relación al diseño no experimental, Hernández, Fernández y Baptista (2014), lo definen como aquellos empleados en las investigaciones enfocadas a realizar descripciones de los fenómenos de estudio, pues las inferencias se realizan sin intervención directa o búsqueda de efectos en el fenómeno analizado y no se manipula la variable. Por tanto el investigador, en el contexto de las instituciones investigadas pertenecientes al municipio rural Zona Bananera, departamento Magdalena - Colombia, sólo tomará las muestras tomando distancia de los hechos que se suscitan, a bien de no influir en la evolución del fenómeno.

En cuanto a la clasificación del diseño transeccional, Hernández y otros (2014, p.154), le atribuyen la cualidad de ser "investigaciones que recopilan datos en un momento único"; razón por la cual no pretenden evaluar la evolución del comportamiento de la 
variable, sino que se enfocan en describir variables y analizar su interrelación en un momento dado.

En lo que corresponde al diseño de campo, Arias (2012,p.31), señala que este "consiste en la recolección de datos directamente de los sujetos investigados, o de la realidad donde ocurren los hechos (datos primarios)"; de tal manera, se profundizó el objeto de estudio, pues el investigador tendrá contacto directo con rectores y docentes de instituciones de educación básica; es decir, los datos serán tomados del lugar donde se generan.

La determinación de las técnicas de análisis, guarda relación con la selección de los medios y procedimientos a través de los cuales se extraen de los datos la información pertinente para elaborar respuestas a los cuestionamientos de la investigación; además, tal selección amerita mantener correspondencia entre las pruebas estadísticas y el tipo de investigación desarrollada. Es decir, que el análisis de los datos en este estudio, se realizó aplicando la estadística inferencial, concretamente el análisis de la varianza ANOVA de un factor de alternativas seleccionadas por los sujetos de la investigación en el instrumento de recolección de datos y posterior ubicación en baremo.

Al efecto, de acuerdo al criterio de Arias (2012) se consideró el total de datos que resulta de multiplicar el número de sujetos por el número de ítems de cada indicador. Ahora bien, para la categorización de los valores presentados con la calificación, se realizó el siguiente baremo, el cual responde a la construcción siguiendo los métodos estadísticos para de esa manera determinar su ubicación.

\section{DERIVACIONES CONCLUSIVAS DE LA INVESTIGACIÓN}

Seguidamente, se presentan las conclusiones sobre la base de los hallazgos obtenidos en los resultados concernientes a al estudio. En este sentido, se determinaron las capacidades tecnológicas empleadas por los docentes de las instituciones educativas del Municipio Zona Bananera, Magdalena - Colombia, se revelaron debilidades en 
directivos y docentes, pues los indicadores tienen poca presencia en la dimensión capacidades tecnológicas, concretamente con el manejo de programas, aprovechamiento de la información y manejo de la internet, siendo desfavorable para impulsar proyectos en correspondencia con las necesidades de autogestión y de aprendizaje en los estudiantes. Esto a su vez, imposibilitará el surgimiento de procesos novedosos, acciones creativas y planificaciones estratégicas encaminadas a la dinamización de la praxis gerencial y del aula de clase.

\section{Recomendaciones de la investigación}

Las recomendaciones se orientan a partir de algunos aspectos que posibilitan acercarse favorablemente a la problemática, considerando acciones oportunas para coadyuvar a la problemática abordada. Promover la reorientación de la gestión de la filosofía institución hacia la construcción de experiencias significativas con el desarrollo de proyectos productivos impulsados desde la gerencia al aula de clase, donde oportunamente cada docente renovará sus acciones en aras de mejorar la responsabilidad, corresponsabilidad y su participación en cada de las actividades asociadas con las TIC. En relación a lo anteriormente expresado es importante señalar lo que plantea Colina (2018):

...es imprescindible conocer la nueva visión de los roles que desempeñan los docentes y los estudiantes, donde el profesor deja de ser el protagonista, es decir, la acción docente que se desarrolla se fundamenta en el principio de responsabilidad compartida entre el participante y el facilitador, convirtiéndose en un mediador del proceso educativo y le cede el paso al estudiante, el cual, debe tener un compromiso firme con su propio proceso de formación. Debido a esto, es la necesidad de una planificación didáctica, donde se organizan los momentos y estrategias pedagógicas que interviene el proceso de aprendizaje - enseñanza, tanto en las horas presénciales como las horas de trabajo independiente ( $p$ 94).

En este sentido, las reuniones, círculos de formación constructiva y encuentros de pares, fomentarán una cultura basada en la moral, ética y responsabilidad hacia cada uno de los proyectos impulsados con orientación endógena, a bien de la obtención de 
beneficios económicos. Apoyar en todo momento, las iniciativas de docentes a través de la aplicación de las fases del uso de las TIC, para ello es necesario tener un conocimiento en planificación estratégica de la realidad socio-contextual de la escuela, por tanto se requiere participar en encuentros quincenales planificados por la gerencia para el debatir de ideas y opiniones acerca de las gestiones en materia socioproductiva, pero sobre todo impulsar procesos de formación permanente en el personal orientado a la construcción de un conocimiento tecnológico que permita operativizar las actividades y las tareas de los proyectos.

\section{REFERENCIAS CONSULTADAS}

1. Aldape, T. (2008). Desarrollo de las competencias del Docente demanda de la aldea global siglo XXI. Libros en red. Recuperado de http://es.slideshare.net/maribelramirezfalcon/edesarrollodelascompetenciasdocen tes.

2. Ander-Egg, E. (2004). Metodología y práctica del desarrollo de la comunidad. Buenos Aires: Lumen/ Humanistas. Buenos Aires.

3. Arias, F. (2012). El proyecto de Investigación. Introducción a la metodología científica. Editorial Episteme. 5ta Edición.

4. Cadenas, H. (2011). La función del funcionalismo: una exploración conceptual. Sociologías, 18(41), 196-214.

5. Colina, M. (2018). AMBIENTE VIRTUAL DE APRENDIZAJE DE CONTABILIDAD II PARA LAS HORAS DE TRABAJO INDEPENDIENTE DEL PROGRAMA NACIONAL DE FORMACIÓN EN ADMINISTRACIÓN. Revista Arbitrada Interdisciplinaria Koinonía, 2(3), 92-108. Recuperado de http://fundacionkoinonia.com.ve/ojs/index.php/revistakoinonia/article/view/54

6. Fernández, J. (2009). Elementos que consolidan el concepto profesión. Revista Electrónica de Investigación Educativa, 3(2), 1-8. Recuperado de http://redie.uabc.mx/redie/article/view/40/1180.

7. Guzmán, A. y Briceño, M. (2010). Teaching competencies for technology integration in the classroom. Journal of Computer Assisted Learning, 20, 453469. 
8. Hernández, R., Fernández, C. y Baptista, L. (2014). Metodología de la investigación. Edición 4ra.

9. Hurtado, I. (2010). Paradigmas y métodos de investigación en tiempos de cambio. Venezuela. Editorial CEC.

10.Luthans, F (2006) Comportamiento organizacional. Ed. Mcgrawill Interamericana Editores. S.A.

11. Marquéz, P. (2010). Impacto de las TIC en educación: funciones y limitaciones. Universidad Nacional Abierta. Documento en línea, disponible en: http://dewey.uab.es/pmarques/siyedu.htm.

12. Oyarzo, R (2010) Evaluación de las herramientas tecnológicas para el aprendizaje del inglés en la UTE y la percepción de los estudiantes. Revista electrónica Revista EÍDOS. Universidad Tecnológica Equinoccial. Quito, Ecuador.

13. Rey (2015), Gestión del territorio para usos agropecuarios (GESTUA). Bases para la formulación de política pública, Bogotá, Unidad de Planificación Rural Agropecuaria, Ministerio de Agricultura y Desarrollo Rural.

14. Ruiz D y Cadenas C. (2007) ¿Que en una política pública?. Universidad latina de América. Revista jurídica. http://www.unla.mx/iusunla18/reflexion/QUE\%20ES\%20UNA\%20POLITICA\%20 PUBLICA\%20web.htm.

15.Suarez, C. (2009). Los entornos virtuales de aprendizaje como instrumento de mediación. Universidad de Salamanca. España.

16. Villareal, E (2010) "Políticas Públicas" en Eduardo Villareal Cantú y Víctor Hugo Martínez González (coord.), (Pre) textos para el Análisis Político. Disciplinas, Reglas y Procesos, México, Flacso México, Universidad Von Humbolt, pp. 257279.

(C2019 por el autor. Este artículo es de acceso abierto y distribuido según los términos y condiciones de la licencia Creative Commons Attribution (http://creativecommons.org/licenses/by/4.0/). 